\title{
Independent Sets in Tensor Products of Three Vertex-transitive Graphs
}

\author{
Huiqun Mao and Huajun Zhang*
}

Abstract. The tensor product $T\left(G_{1}, G_{2}, G_{3}\right)$ of graphs $G_{1}, G_{2}$ and $G_{3}$ is defined by

$$
V T\left(G_{1}, G_{2}, G_{3}\right)=V\left(G_{1}\right) \times V\left(G_{2}\right) \times V\left(G_{3}\right)
$$

and

$$
E T\left(G_{1}, G_{2}, G_{3}\right)=\left\{\left[\left(u_{1}, u_{2}, u_{3}\right),\left(v_{1}, v_{2}, v_{3}\right)\right]:\left|\left\{i:\left(u_{i}, v_{i}\right) \in E\left(G_{i}\right)\right\}\right| \geq 2\right\} .
$$

From this definition, it is easy to see that the preimage of the direct product of two independent sets of two factors under projections is an independent set of $T\left(G_{1}, G_{2}, G_{3}\right)$. So

$$
\alpha T\left(G_{1}, G_{2}, G_{3}\right) \geq \max \left\{\alpha\left(G_{1}\right) \alpha\left(G_{2}\right)\left|G_{3}\right|, \alpha\left(G_{1}\right) \alpha\left(G_{3}\right)\left|G_{2}\right|, \alpha\left(G_{2}\right) \alpha\left(G_{3}\right)\left|G_{1}\right|\right\} .
$$

In this paper, we prove that the equality holds if $G_{1}$ and $G_{2}$ are vertex-transitive graphs and $G_{3}$ is a circular graph, a Kneser graph, or a permutation graph. Furthermore, in this case, the structure of all maximum independent sets of $T\left(G_{1}, G_{2}, G_{3}\right)$ is determined.

\section{Introduction}

Let $G$ and $H$ be two graphs. The direct product $G \times H$ of $G$ and $H$ is defined by

$$
V(G \times H)=V(G) \times V(H)=\{(a, u): a \in V(G) \text { and } u \in V(H)\}
$$

and

$$
E(G \times H)=\{[(a, u),(b, v)]:(a, b) \in E(G) \text { and }(u, v) \in E(H)\} .
$$

It is an interesting problem to determine the independence number of the direct product of two graphs $G$ and $H$. Clearly, the preimage of $I$ under projections is an independent set of $G \times H$ if $I$ is an independent set of $G$ or $H$. Hence, $\alpha(G \times H) \geq \max \{\alpha(G)|H|, \alpha(H)|G|\}$,

Received April 9, 2019; Accepted October 11, 2020.

Communicated by Sen-Peng Eu.

2020 Mathematics Subject Classification. 05D05, 06A07.

Key words and phrases. direct product, tensor product, primitivity, independence number, vertex-transitive graph.

Zhang was partially supported by the National Natural Science Foundation of China (Nos. 11971439 and 12031018).

*Corresponding author. 
where $\alpha(G)$ denotes the independence number of $G$ and $|G|$ denotes the order of $G$. A natural question is whether the equality holds or not. Jha and Klavžar [6] proved that the equality cannot always hold if one of the two graphs is non-vertex-transitive, where a graph $G$ is said to be vertex-transitive if for every pair vertices $u$ and $v$ of $G$, there exists $f$ of the automorphism group of $G$ such that $f(u)=v$. So Tardif [11 proposed the following problem.

Problem 1.1. (Tardif 11]) Does

$$
\alpha(G \times H)=\max \{\alpha(G)|H|, \alpha(H)|G|\}
$$

hold for vertex-transitive graphs $G$ and $H$ ?

Furthermore, if the equality holds, then there is another interesting problem as follows.

Problem 1.2. If $\alpha(G \times H)=\max \{\alpha(G)|H|, \alpha(H)|G|\}$, is every maximum independent set of $G \times H$ the preimage of an independent set of one factor under projections?

An independent set of $G \times H$ is called regular if it is the preimage of an independent set of one factor under projections. We say that the direct product $G \times H$ is MIS-normal (maximum-independent-set-normal) if every maximum independent set of $G \times H$ is regular.

There are many results on these two problems. Frankl [4] and Valencia-Pabon and Vera 12 solved Problem 1.1 for Kneser graphs and circular graphs, respectively. Larose and Tardif [10] characterized the structures of maximum independent sets in powers of circular graphs, Kneser graphs and truncated simplices. $\mathrm{Ku}$ and Wong [9] showed the structure of maximum independent sets in direct products of permutation graphs. Wang and $\mathrm{Yu}$ [14] proved that both Problems 1.1 and 1.2 have positive answers if one of $G$ and $H$ is a bipartite graph. Recently, Zhang [17] gave an affirmative answer to Problem 1.1 and showed the structure of maximum independent sets in direct products of vertex-transitive graphs $G$ and $H$. (See also $1,5,8,16$ for related results on Problems 1.1 and 1.2.)

For a graph $G$, let $S(G)$ denote the set of all independent sets of $G$. A fractional coloring of $G$ is a mapping $f$ from $S(G)$ to $[0,1]$ such that $\sum_{u \in S \in S(G)} f(S)=1$ for every $u \in V(G)$. The total weight $w(f)$ of a fractional coloring $f$ of $G$ is defined by $w(f)=\sum_{S \in S(G)} f(S)$. We call the minimum total weight of a fractional coloring of $G$ the fractional chromatic number $\chi_{f}(G)$ of $G$. It is well-known that $\chi_{f}(G)=|G| / \alpha(G)$ if $G$ is vertex-transitive. In 18, Zhu proved that the equality $\chi_{f}(G \times H)=\min \left\{\chi_{f}(G), \chi_{f}(H)\right\}$ holds for all graphs $G$ and $H$.

Before stating the results, we introduce some notation. For a graph $G$, let $I(G)$ denote the set of all maximum independent sets of $G$. Given a subset $A$ of $V(G)$, define

$$
\begin{aligned}
& N_{G}(A)=\{b \in V(G):(a, b) \in E(G) \text { for some } a \in A\}, \\
& N_{G}[A]=N_{G}(A) \cup A \text { and } \bar{N}_{G}[A]=V(G) \backslash N_{G}[A] .
\end{aligned}
$$


The following two results, which can be deduced from the so-called "No-Homomorphism" lemma of Albertson and Collins [2], are useful to get bounds on the sizes of independent sets.

Lemma 1.3. (Cameron and $\mathrm{Ku}[3]$ ) Let $G$ be a vertex-transitive graph and $A \subseteq V(G)$. Then $\frac{\alpha(G[A])}{|A|} \geq \frac{\alpha(G)}{|G|}$. The equality implies that $|S \cap A|=\alpha(G[A])$ for every maximum independent set $S \in I(G)$.

Lemma 1.4. (Zhang [16]) Let $G$ be a vertex-transitive graph. Then, for every independent set $A$ of $G, \frac{|A|}{\left|N_{G}[A]\right|} \leq \frac{\alpha(G)}{|G|}$. The equality implies that $\left|S \cap N_{G}[A]\right|=|A|$ for every $S \in I(G)$, and in particular $A \subseteq S$ for some $S \in I(G)$.

An independent set $A$ in a graph $G$ is said to be imprimitive if $|A|<\alpha(G)$ and $\frac{|A|}{\left|N_{G}[A]\right|}=$ $\frac{\alpha(G)}{|V(G)|}$. The graph $G$ is called $I S$-imprimitive if $G$ has an imprimitive independent set. Otherwise, $G$ is called IS-primitive. Clearly, a vertex-transitive graph is IS-imprimitive if it is disconnected. So an IS-primitive vertex-transitive graph must be connected. But an IS-imprimitive graph is not necessarily disconnected. In [17], Zhang obtained the following result.

Theorem 1.5. (Zhang $|17|)$ Let $G$ and $H$ be two vertex-transitive graphs with $\frac{\alpha(G)}{|G|} \geq$ $\frac{\alpha(H)}{|H|}$. Then

$$
\alpha(G \times H)=\alpha(G)|H|
$$

and exactly one of the following holds:

(i) $G \times H$ is MIS-normal,

(ii) $\frac{\alpha(G)}{|G|}=\frac{\alpha(H)}{|H|}$ and one of $G$ or $H$ is IS-imprimitive,

(iii) $\frac{\alpha(G)}{|G|}>\frac{\alpha(H)}{|H|}$ and $H$ is disconnected.

Note that when condition (ii) holds, the direct product $G \times H$ is not MIS-normal. In fact, if $A$ is an imprimitive set of $G$, then $S=A \times V(H) \cup \bar{N}_{G}[A] \times I$ is an independent set of $G \times H$ and $|S|=\alpha(G)|H|$, where $I \in I(H)$. It is clear that the direct product $G \times H$ is not MIS-normal when condition (iii) holds.

For three graphs $G_{1}, G_{2}$ and $G_{3}$, their direct products $G_{1} \times G_{2} \times G_{3}$ with vertex set $V\left(G_{1}\right) \times V\left(G_{2}\right) \times V\left(G_{3}\right)$ and edge set

$$
E\left(G_{1} \times G_{2} \times G_{3}\right)=\left\{\left[\left(u_{1}, u_{2}, u_{3}\right),\left(v_{1}, v_{2}, v_{3}\right)\right]:\left(u_{i}, v_{i}\right) \in E\left(G_{i}\right)\right\} .
$$

Recently, Tardif generalized it and gave the definition of tensor product $T\left(G_{1}, G_{2}, G_{3}\right)$ of $G_{1}, G_{2}$ and $G_{3}$, which with the same vertex set as $G_{1} \times G_{2} \times G_{3}$ and edge set

$$
E T\left(G_{1}, G_{2}, G_{3}\right)=\left\{\left[\left(u_{1}, u_{2}, u_{3}\right),\left(v_{1}, v_{2}, v_{3}\right)\right]:\left|\left\{i:\left(u_{i}, v_{i}\right) \in E\left(G_{i}\right)\right\}\right| \geq 2\right\} .
$$


Clearly, $E\left(G_{1} \times G_{2} \times G_{3}\right) \subseteq E T\left(G_{1}, G_{2}, G_{3}\right)$ and the tensor product is also commutative and associative.

For $i=1,2,3$ and $A_{i} \subseteq V\left(G_{i}\right)$, let $A_{1} \times A_{2} \times A_{3}=\left\{\left(u_{1}, u_{2}, u_{3}\right): u_{i} \in A_{i}\right\}$. Clearly, $I_{1} \times I_{2} \times G_{3}, I_{1} \times G_{2} \times I_{3}$ and $G_{1} \times I_{2} \times I_{3}$ are independent sets of $\left(G_{1}, G_{2}, G_{3}\right)$ if $I_{i}$ is an independent set of $G_{i}, i=1,2,3$. So the inequality

$$
\alpha T\left(G_{1}, G_{2}, G_{3}\right) \geq \max \left\{\alpha\left(G_{1}\right) \alpha\left(G_{2}\right)\left|G_{3}\right|, \alpha\left(G_{1}\right) \alpha\left(G_{3}\right)\left|G_{2}\right|, \alpha\left(G_{2}\right) \alpha\left(G_{3}\right)\left|G_{1}\right|\right\}
$$

holds. It is natural to ask the following problem.

Problem 1.6. For three vertex-transitive graphs $G_{1}, G_{2}$ and $G_{3}$, does

$$
\alpha T\left(G_{1}, G_{2}, G_{3}\right)=\max \left\{\alpha\left(G_{1}\right) \alpha\left(G_{2}\right)\left|G_{3}\right|, \alpha\left(G_{1}\right) \alpha\left(G_{3}\right)\left|G_{2}\right|, \alpha\left(G_{2}\right) \alpha\left(G_{3}\right)\left|G_{1}\right|\right\}
$$

hold? Furthermore, if the equality holds, is every maximum independent set of $T\left(G_{1}, G_{2}\right.$, $\left.G_{3}\right)$ the preimage of the direct product of two independent sets of two factors under projections?

In fact, the equality (1.1) does not always hold for non-vertex-transitive $G_{1}, G_{2}$ and $G_{3}$. For example, in Figure 1.1, let $S=\left\{v_{1}\right\} \times\left\{v_{9}\right\} \times\left\{v_{10}, v_{11}, v_{12}\right\} \cup\left\{v_{1}\right\} \times\left\{v_{5}, v_{6}, v_{7}, v_{8}\right\} \times$ $\left\{v_{10}\right\}$. Clearly, $\alpha\left(G_{1}\right)=1, \alpha\left(G_{2}\right)=2, \alpha\left(G_{3}\right)=1$ and $\max \left\{\alpha\left(G_{1}\right) \alpha\left(G_{2}\right)\left|G_{3}\right|, \alpha\left(G_{1}\right) \alpha\left(G_{3}\right)\right.$ $\left.\left|G_{2}\right|, \alpha\left(G_{2}\right) \alpha\left(G_{3}\right)\left|G_{1}\right|\right\}=6$. But $S$ is an independent set of $T\left(G_{1}, G_{2}, G_{3}\right)$ of size 7 .

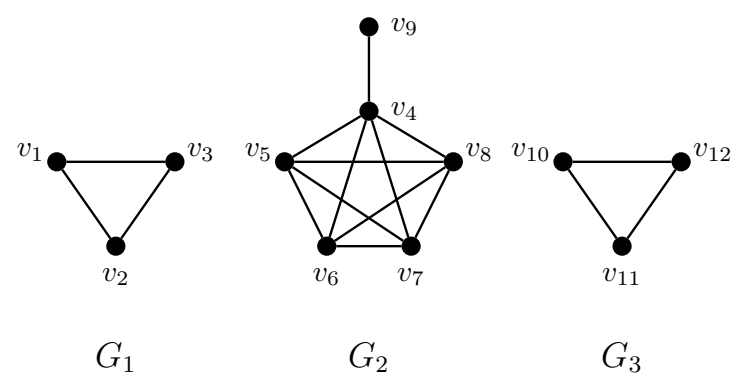

Figure 1.1: Graphs $G_{1}, G_{2}, G_{3}$.

Similarly, we say that an independent set of $T\left(G_{1}, G_{2}, G_{3}\right)$ is regular if it is the preimage of the direct product of two independent sets of two factors under projections, and the tensor product $T\left(G_{1}, G_{2}, G_{3}\right)$ is called MIS-normal if every maximum independent set of $T\left(G_{1}, G_{2}, G_{3}\right)$ is regular.

Let $n \geq 2 r$. The well-known circular graph $K_{n: r}$ has the vertex set $[n]=\{1,2, \ldots, n\}$, and for any $i, j \in[n]$, they are adjacent if and only if $r \leq|i-j| \leq n-r$. It is easy to see that $K_{n: r}$ is vertex-transitive, and $\alpha\left(K_{n: r}\right)=r$ by the well-known result of Katona [7]. 
In [15, the authors proved that the equality

$$
\chi_{f} T\left(G, H, K_{n: r}\right)=\min \left\{n \chi_{f}(G) / r, n \chi_{f}(H) / r, \chi_{f}(G) \chi_{f}(H)\right\}
$$

holds for all graphs $G$ and $H$. That is, the equality (1.1) holds if one of $G_{1}, G_{2}$ and $G_{3}$ is a circular graph.

In this paper, we will characterized the structures of maximum independent sets of $T\left(G_{1}, G_{2}, G_{3}\right)$ if one of $G_{1}, G_{2}$ and $G_{3}$ is a circular graph.

The following theorem is the main result of this paper.

Theorem 1.7. Let $G$ and $H$ be two vertex-transitive graphs with $\frac{\alpha(G)}{|G|} \geq \frac{\alpha(H)}{|H|}$ and $K_{n: r}$ be a circular graph. Then

$$
\alpha T\left(G, H, K_{n: r}\right)=\max \{\alpha(G) \alpha(H) n, \alpha(G) r|H|\}
$$

and exactly one of the following holds:

(i) $T\left(G_{1}, G_{2}, G_{3}\right)$ is MIS-normal,

(ii) $\min \left\{\frac{r}{n}, \frac{\alpha(G)}{|G|}\right\}>\frac{\alpha(H)}{|H|}$ and $H$ is disconnected,

(iii) $\frac{r}{n}>\frac{\alpha(H)}{|H|}=\frac{\alpha(G)}{|G|}$ and $G$ or $H$ is IS-imprimitive,

(iv) $\frac{\alpha(G)}{|G|}>\frac{r}{n}=\frac{\alpha(H)}{|H|}$ and $H$ is IS-imprimitive,

(v) $\frac{r}{n}=\frac{\alpha(H)}{|H|}=\frac{\alpha(G)}{|G|}$ and one of them is IS-imprimitive.

Similarly, it is clear that $T\left(G_{1}, G_{2}, G_{3}\right)$ is not MIS-normal when one of conditions (ii)(v) holds. Indeed, when $\max \left\{\frac{\alpha\left(G_{1}\right)}{\left|G_{1}\right|}, \frac{\alpha\left(G_{2}\right)}{\left|G_{2}\right|}\right\} \leq \frac{\alpha\left(G_{3}\right)}{\left|G_{3}\right|}, T \times I_{3}$ is a maximum independent set of $T\left(G_{1}, G_{2}, G_{3}\right)$ if $T$ is a maximum independent set of $G_{1} \times G_{2}$, where $I_{3}$ is a maximum independent set of $G_{3}$.

The rest of this paper is organized as follows. In the next section, we present some preliminary results. Then we will prove Theorem 1.7 and give some results on Kneser graphs and permutation graphs in Section 3.

\section{Preliminary results}

Let $A \subseteq V(G), U \subseteq V(H), I \subseteq V\left(K_{n: r}\right)$, and $S$ be a maximum independent set of $T\left(G, H, K_{n: r}\right)$. For simplicity, let $(a, u) \times I=\bigcup_{i \in I}(a, u, i)$ for $a \in A$ and $u \in U$. Define

$$
\partial_{a, u}(S)=\{i \in[n]:(a, u, i) \in S\}
$$


for $a \in G$ and $u \in H$. In the sequel, let $I$ be a fixed maximum independent set of $K_{n: r}$. $\triangle(S)$ is defined as a subset of $V T\left(G_{1}, G_{2}, G_{3}\right)$ such that, for $(a, u) \in V(G) \times V(H)$, $\partial_{a, u}(\triangle(S))=I$ if $0<\left|\partial_{a, u}(S)\right|<n$ and $\partial_{a, u}(\triangle(S))=\partial_{a, u}(S)$ otherwise. Clearly,

$$
\triangle(S)=\left\{(a, u) \times I: 0<\left|\partial_{a, u}(S)\right|<n\right\} \cup\left\{(a, u) \times[n]:\left|\partial_{a, u}(S)\right|=n\right\},
$$

and $\triangle(\triangle(S))=\triangle(S)$. In the following, we will prove that $\triangle(S)$ is also a maximum independent set of $\left(G, H, K_{n: r}\right)$. We first introduce a result in [5].

Lemma 2.1. (Geng et al. [5]) Let $J_{1}, J_{2} \subseteq V\left(K_{n: r}\right)$. If $\left(j_{1}, j_{2}\right) \notin E\left(K_{n: r}\right)$ for every pair $j_{1} \in J_{1}$ and $j_{2} \in J_{2}$, then $\left|J_{1}\right|+\left|J_{2}\right| \leq 2 r$.

Lemma 2.2. Let $S$ be a maximum independent set of $T\left(G_{1}, G_{2}, G_{3}\right)$. Then $\triangle(S)$ is also a maximum independent set of $T\left(G_{1}, G_{2}, G_{3}\right)$.

Proof. Let

$$
\begin{gathered}
\partial_{G, H}(S)=\left\{(a, u) \in V(G) \times V(H): \partial_{a, u}(S) \neq \emptyset\right\}, \\
\mathcal{D}_{1}=\left\{(a, u) \in \partial_{G, H}(S):\left|\partial_{a, u}(S)\right|=n\right\}, \\
\mathcal{D}_{2}=\left\{(a, u) \in \partial_{G, H}(S): r<\left|\partial_{a, u}(S)\right|<n\right\}
\end{gathered}
$$

and

$$
\mathcal{D}_{3}=\left\{(a, u) \in \partial_{G, H}(S): 0<\left|\partial_{a, u}(S)\right| \leq r\right\}
$$

Then,

$$
\triangle(S)=\left\{(a, u) \times[n]:(a, u) \in \mathcal{D}_{1}\right\} \cup\left\{(a, u) \times I:(a, u) \in \mathcal{D}_{2} \cup \mathcal{D}_{3}\right\} .
$$

For $(a, u),(b, v) \in V(G \times H)$, if $(a, b) \in E(G)$ or $(u, v) \in E(H)$, it follows by definition that $(i, j) \notin E\left(K_{n: r}\right)$ for all $i \in \partial_{a, u}(S)$ and $j \in \partial_{b, v}(S)$, and then Lemma 2.1 implies $\left|\partial_{a, u}(S)\right|+\left|\partial_{b, v}(S)\right| \leq 2 r$. Therefore, by the definitions of $\mathcal{D}_{1}, \mathcal{D}_{2}$ and $\mathcal{D}_{3}$, it follows that $(a, b) \notin E(G)$ and $(u, v) \notin E(H)$ for $(a, u),(b, v) \in \mathcal{D}_{1} \cup \mathcal{D}_{2}$ or $(a, u) \in \mathcal{D}_{1}$ and $(b, v) \in \mathcal{D}_{3}$. Hence $\triangle(S)$ is an independent set of $T\left(G_{1}, G_{2}, G_{3}\right)$.

Now we prove that $|\triangle(S)|=|S|$. If $\mathcal{D}_{2} \cup \mathcal{D}_{3}=\emptyset$, it is clear that $\triangle(S)=S$ and then the result holds. So we assume that $\mathcal{D}_{2} \cup \mathcal{D}_{3} \neq \emptyset$. If $\mathcal{D}_{3}=\emptyset$, then

$$
\mathcal{S}^{\prime}=\left\{(a, u) \times[n]:(a, u) \in \mathcal{D}_{1} \cup \mathcal{D}_{2}\right\}
$$

is also an independent set of $T\left(G_{1}, G_{2}, G_{3}\right)$ with $\left|S^{\prime}\right|>|S|$, yielding a contradiction. Therefore, $\mathcal{D}_{3} \neq \emptyset$. If $\mathcal{D}_{2}=\emptyset$, then it follows by the definition of $\triangle(S)$ that $|\triangle(S)| \geq|S|$, and the maximality of $S$ further implies $|\triangle(S)|=|S|$. Hence it remains to verify the case $\mathcal{D}_{2} \neq \emptyset$ and $\mathcal{D}_{3} \neq \emptyset$.

For any $(a, u) \in \mathcal{D}_{2}$, if $(a, b) \notin E(G)$ and $(u, v) \notin E(H)$ for all $(b, v) \in \mathcal{D}_{3}$, then

$$
S^{\prime \prime}=S \cup(a, u) \times[n]
$$


is an independent set of $T\left(G_{1}, G_{2}, G_{3}\right)$ with $\left|S^{\prime \prime}\right|>|S|$, a contradiction. So there exists at least one element $(b, v) \in \mathcal{D}_{3}$ such that either $(a, b) \in E(G)$ or $(u, v) \in E(H)$. For $(a, u) \in$ $\mathcal{D}_{2}$, let $N_{\mathcal{D}_{3}}(a, u)$ be the set of all $(b, v) \in \mathcal{D}_{3}$ with either $(a, b) \in E(G)$ or $(u, v) \in E(H)$, and for $\mathcal{D} \subseteq \mathcal{D}_{2}$, set $N_{\mathcal{D}_{3}}(\mathcal{D})=\bigcup_{(a, u) \in \mathcal{D}} N_{\mathcal{D}_{3}}(a, u)$. For any $(b, v) \in N_{\mathcal{D}_{3}}\left(\mathcal{D}_{2}\right)$, there exists an $(a, u) \in \mathcal{D}_{2}$ such that either $(a, b) \in E(G)$ or $(u, v) \in E(H)$. Then it follows from Lemma 2.1 that

$$
\left|\partial_{a, u}(S)\right|+\left|\partial_{b, v}(S)\right| \leq 2 r
$$

and hence

$$
\left|\partial_{b, v}(S)\right|<r
$$

Let $t$ be the largest positive integer such that $\left|N_{\mathcal{D}_{3}}(\mathcal{D})\right| \geq|\mathcal{D}|$ holds for all $\mathcal{D} \subseteq$ $\mathcal{D}_{2}$ whenever $|\mathcal{D}| \leq t$. We now prove that $\left|\mathcal{D}_{2}\right|=t$. If $\left|\mathcal{D}_{2}\right|>t$, then there exists a $(t+1)$-subset $\mathcal{D}=\left\{\left(a_{1}, u_{1}\right),\left(a_{2}, u_{2}\right), \ldots,\left(a_{t+1}, u_{t+1}\right)\right\}$ of $\mathcal{D}_{2}$ such that $N_{\mathcal{D}_{3}}(\mathcal{D})=$ $\left\{\left(b_{1}, v_{1}\right),\left(b_{2}, v_{2}\right), \ldots,\left(b_{t}, v_{t}\right)\right\}$. Set

$$
S^{\prime \prime \prime}=\left(\bigcup_{i=1}^{t+1}\left(a_{i}, u_{i}\right) \times\left([n] \backslash \partial_{a_{i}, u_{i}}(S)\right)\right) \cup\left(S \backslash \bigcup_{j=1}^{t}\left(b_{j}, v_{j}\right) \times \partial_{b_{j}, v_{j}}(S)\right) .
$$

It is easy to verify that $S^{\prime \prime \prime}$ is also an independent set of $T\left(G_{1}, G_{2}, G_{3}\right)$. By the wellknown Hall's marriage theorem, we may reorder the elements of $N_{\mathcal{D}_{3}}(\mathcal{D})$ such that either $\left(a_{i}, b_{i}\right) \in E(G)$ or $\left(u_{i}, v_{i}\right) \in E(H)$ for $1 \leq i \leq t$. Then, by Lemma 2.1 and $n \geq 2 r$, we have that

$$
\begin{aligned}
\left|S^{\prime \prime \prime}\right|-|S| & =\bigcup_{i=1}^{t+1}\left|\left(a_{i}, u_{i}\right) \times\left([n] \backslash \partial_{a_{i}, u_{i}}(S)\right)\right|-\bigcup_{j=1}^{t}\left|\left(b_{j}, v_{j}\right) \times \partial_{b_{j}, v_{j}}(S)\right| \\
& =\sum_{i=1}^{t}\left(n-\left|\partial_{a_{i}, u_{i}}(S)\right|-\left|\partial_{b_{i}, v_{i}}(S)\right|\right)+\left(n-\left|\partial_{a_{t+1}, u_{t+1}}(S)\right|\right) \\
& \geq \sum_{i=1}^{t}(n-2 r)+\left(n-\left|\partial_{a_{t+1}, u_{t+1}}(S)\right|\right)>0,
\end{aligned}
$$

a contradiction. Hence, $\left|\mathcal{D}_{2}\right|=t$. Now, let $\mathcal{D}_{2}=\left\{\left(a_{1}, u_{1}\right),\left(a_{2}, u_{2}\right), \ldots,\left(a_{t}, u_{t}\right)\right\}$ and $N_{\mathcal{D}_{3}}\left(\mathcal{D}_{2}\right)=\left\{\left(b_{1}, v_{1}\right),\left(b_{2}, v_{2}\right), \ldots,\left(b_{s}, v_{s}\right)\right\}$ such that either $\left(a_{i}, b_{i}\right) \in E(G)$ or $\left(u_{i}, v_{i}\right) \in$ $E(H)$ for $1 \leq i \leq t$, where $s \geq t$. Then, it follows from Lemma 2.1 that

$$
\begin{aligned}
|\triangle(S)|-|S|= & \sum_{(a, u) \in \mathcal{D}_{2}}\left(r-\left|\partial_{a, u}(S)\right|\right)+\sum_{(b, v) \in \mathcal{D}_{3}}\left(r-\left|\partial_{b, v}(S)\right|\right) \\
\geq & \sum_{i=1}^{t}\left(2 r-\left|\partial_{a_{i}, u_{i}}(S)\right|-\left|\partial_{b_{i}, v_{i}}(S)\right|\right)+\sum_{j=t+1}^{s}\left(r-\left|\partial_{b_{j}, v_{j}}(S)\right|\right) \\
& +\sum_{(c, w) \in \mathcal{D}_{3} \backslash N_{\mathcal{D}_{3}}\left(\mathcal{D}_{2}\right)}\left(r-\left|\partial_{c, w}(S)\right|\right)
\end{aligned}
$$




$$
\geq 0
$$

and the maximality of $S$ implies that $|\triangle(S)|=|S|$.

\section{Proof of Theorem 1.7}

We are now ready to prove the main result (Theorem 1.7) in this paper.

Proof of Theorem 1.7. If one of $G, H$ and $K_{n: r}$ is empty graph, the result is obvious. Note that for each vertex-transitive graph $G$, either $\frac{\alpha(G)}{|G|}=1$ or $\frac{\alpha(G)}{|G|} \leq \frac{1}{2}$. So we suppose $\max \left\{\frac{\alpha(G)}{|G|}, \frac{\alpha(H)}{|H|}, \frac{r}{n}\right\} \leq \frac{1}{2}$. Let $T$ be a maximum independent set of $T\left(G_{1}, G_{2}, G_{3}\right)$. Clearly, $|T| \geq \max \{\alpha(G) \alpha(H) n, \alpha(G) r|H|\}$. Let $S=\triangle(T)$, then by Lemma 2.2, $S$ is also a maximum independent set and $|S|=|T|$. Furthermore, by definition, $\triangle(S)=S$. Let $\mathcal{D}_{1}$, $\mathcal{D}_{2}$ and $\mathcal{D}_{3}$ be defined as in Lemma 2.2 according to $S$. Then $\mathcal{D}_{2}=\emptyset$. In the following, we will prove that $|S| \leq \max \{\alpha(G) \alpha(H) n, \alpha(G) r|H|\}$.

Set

$$
A_{1}=\left\{a \in V(G):(a, u) \in \mathcal{D}_{1} \text { for some } u \in V(H)\right\}
$$

and

$$
U_{0}=\left\{u \in V(H):(a, u) \in \mathcal{D}_{1} \text { for some } a \in V(G)\right\} .
$$

Note that $(a, b) \notin E(G)$ and $(u, v) \notin E(H)$ hold for all $(a, u),(b, v) \in \mathcal{D}_{1} \cup \mathcal{D}_{2}$ or $(a, u) \in \mathcal{D}_{1}$ and $(b, v) \in \mathcal{D}_{1} \cup \mathcal{D}_{3}$. Hence, $A_{1}$ and $U_{0}$ are independent sets of $G$ and $H$ respectively. Moreover, we have that

$$
\mathcal{D}_{3} \subseteq \bar{N}_{G}\left[A_{1}\right] \times \bar{N}_{H}\left[U_{0}\right] \cup A_{1} \times \bar{N}_{H}\left[U_{0}\right] \cup \bar{N}_{G}\left[A_{1}\right] \times U_{0}
$$

Furthermore, by the maximality of $S$, we can deduce that

$$
\mathcal{D}_{1}=A_{1} \times U_{0}
$$

and

$$
\bar{N}_{G}\left[A_{1}\right] \times U_{0} \cup A_{1} \times \bar{N}_{H}\left[U_{0}\right] \subseteq \mathcal{D}_{3}
$$

For $u \in V(H)$, define

$$
X_{u}=\left\{a \in V(G):(a, u) \in \mathcal{D}_{3}\right\} .
$$

Let $G\left[X_{u}\right]$ be the subgraph of $G$ induced by $X_{u}$. Consider the decomposition $X_{u}=$ $X_{u}^{*} \cup X_{u}^{\prime}$, where $X_{u}^{*}$ is the set of all isolated vertices in $G\left[X_{u}\right]$. Clearly, $X_{u}^{*}=\emptyset$ and $X_{u}^{\prime}=\bar{N}_{G}\left[A_{1}\right]$ if $u \in U_{0} ; X_{u}=\emptyset$ if $u \in N_{H}\left(U_{0}\right) ; A_{1} \subseteq X_{u}^{*}$ and $X_{u}^{\prime} \subseteq \bar{N}_{G}\left[A_{1}\right]$ if $u \in \bar{N}_{H}\left[U_{0}\right]$. Set $X^{\prime}=\bigcup_{u \in H} X_{u}^{\prime}$, then $X^{\prime} \subseteq \bar{N}_{G}\left[A_{1}\right]$. For $u \in \bar{N}_{H}\left[U_{0}\right], X_{u}^{*}$ is an independent set of $G$. Note that $X_{u}^{*}$ may be equal to $A_{1}$. We list all distinct $X_{u}^{*}$ 's as $A_{2}, \ldots, A_{s}$. 
Let $U_{1}, U_{2}, \ldots, U_{s}$ be defined as follows:

$$
U_{1}=N_{H}\left[U_{0}\right] \quad \text { and } \quad U_{i}=\left\{u \in \bar{N}_{H}\left[U_{0}\right]: X_{u}^{*}=A_{i}\right\}, \quad i=2,3, \ldots, s .
$$

Clearly, $V(H)=U_{1} \cup U_{2} \cup \cdots \cup U_{s}$. By Lemma 1.4, we have that

$$
\frac{\left|U_{0}\right|}{\left|U_{1}\right|}=\frac{\left|U_{0}\right|}{\left|N_{H}\left[U_{0}\right]\right|} \leq \frac{\alpha(H)}{|H|}
$$

For $a \in V(G)$, set

$$
B_{a}=\left\{u \in V(H): a \in X_{u}^{\prime}\right\}
$$

and then

$$
\begin{aligned}
|S| & =\left|\mathcal{D}_{1}\right| n+\left|\mathcal{D}_{3}\right| r=\left|\mathcal{D}_{1}\right| n+\sum_{u \in V(H)}\left|X_{u}\right| r \\
& =\left|A_{1}\right|\left|U_{0}\right| n+\sum_{u \in V(H)}\left|X_{u}^{*}\right| r+\sum_{u \in V(H)}\left|X_{u}^{\prime}\right| r \\
& =\left|A_{1}\right|\left|U_{0}\right| n+\sum_{i=2}^{s}\left|A_{i}\right|\left|U_{i}\right| r+\sum_{u \in V(H)}\left|X_{u}^{\prime}\right| r \\
& =\left|A_{1}\right|\left|U_{0}\right| n+\sum_{i=2}^{s}\left|A_{i}\right|\left|U_{i}\right| r+\sum_{a \in X^{\prime}}\left|B_{a}\right| r .
\end{aligned}
$$

For every pair $u, v \in V(H)$, if $X_{u}^{\prime} \cap X_{v}^{\prime} \neq \emptyset$, then $(u, v) \notin E(H)$. Hence $B_{a}$ is an independent set of $H$. By definition,

$$
B_{a} \subseteq \sum_{i: a \in \bar{N}_{G}\left[A_{i}\right]} U_{i}
$$

Note that $X^{\prime} \subseteq \bar{N}_{G}\left[A_{1}\right]$. We now prove that $U_{i} \subseteq \bar{N}_{H}\left[B_{a}\right]$ if $a \in N_{G}\left[A_{i}\right]$ for $2 \leq i \leq s$, and so $N_{H}\left[B_{a}\right] \subseteq \sum_{i: a \in \bar{N}_{G}\left[A_{i}\right]} U_{i}$. Indeed, suppose that $B_{a} \neq \emptyset$ for some $a \in N_{G}\left[A_{i}\right]$, $2 \leq i \leq s$. It is clear that $U_{i} \cap B_{a}=\emptyset$, hence it remains to verify that $U_{i} \cap N_{H}\left(B_{a}\right)=\emptyset$. If $a \in N_{G}\left(A_{i}\right)$, then $(a, b) \in E(G)$ for some $b \in A_{i}$. Hence, $(a, u) \times I \subseteq S$ and $(b, v) \times I \subseteq S$ for all $u \in B_{a}$ and $v \in U_{i}$. Then it follows by definition that $(u, v) \notin E(H)$, and so $U_{i} \cap N_{H}\left(B_{a}\right)=\emptyset$. If $a \in A_{i}$ and $U_{i} \cap N_{H}\left(B_{a}\right) \neq \emptyset$, then $(u, v) \in E(H)$ for some $u \in U_{i}$ and $v \in B_{a}$. On the other hand, since $a \in X_{v}^{\prime}$, it follows that $(a, c) \in E(G)$ for some $c \in X_{v}^{\prime}$. Hence $(a, u) \times I,(c, v) \times I \subseteq S$. However, $(a, c) \in E(G)$ and $(u, v) \in E(H)$, contradicting that $S$ is an independent set of $T\left(G_{1}, G_{2}, G_{3}\right)$. Therefore, $U_{i} \cap N_{H}\left(B_{a}\right)=\emptyset$, and the result holds. Hence by Lemma 1.4, it follows that

$$
\frac{\left|B_{a}\right|}{\left|\sum_{i: a \in \bar{N}_{G}\left[A_{i}\right]} U_{i}\right|} \leq \frac{\left|B_{a}\right|}{\left|N_{H}\left[B_{a}\right]\right|} \leq \frac{\alpha(H)}{|H|} .
$$


By (3.2), (3.3) and Lemma 1.3 , we have that

$$
\begin{aligned}
|S| & =\left|A_{1}\right|\left|U_{0}\right| n+\sum_{i=2}^{s}\left|A_{i}\right|\left|U_{i}\right| r+\sum_{a \in X^{\prime}}\left|B_{a}\right| r \\
& \leq\left|A_{1}\right|\left|U_{0}\right| n+\sum_{i=2}^{s}\left|A_{i}\right|\left|U_{i}\right| r+\frac{\alpha(H)}{|H|} \sum_{a \in X^{\prime}} \sum_{i: a \in \bar{N}_{G}\left[A_{i}\right]}\left|U_{i}\right| r \\
& =\left|A_{1}\right|\left|U_{0}\right| n+\sum_{i=2}^{s}\left|A_{i}\right|\left|U_{i}\right| r+\frac{\alpha(H)}{|H|} \sum_{i=1}^{s}\left|\bar{N}_{G}\left[A_{i}\right]\right|\left|U_{i}\right| r \\
& \leq\left|A_{1}\right|\left|U_{0}\right| n+\sum_{i=2}^{s}\left|A_{i}\right|\left|U_{i}\right| r+\frac{\alpha(G)}{|G|} \sum_{i=1}^{s}\left|\bar{N}_{G}\left[A_{i}\right]\right|\left|U_{i}\right| r \\
& \leq\left(\left|A_{1}\right|\left|U_{0}\right| n+\alpha\left(\bar{N}_{G}\left[A_{1}\right]\right)\left|U_{1}\right| r\right)+\sum_{i=2}^{s}\left(\left|A_{i}\right|+\alpha\left(\bar{N}_{G}\left[A_{i}\right]\right)\right)\left|U_{i}\right| r \\
& \leq\left(\left|A_{1}\right|\left|U_{0}\right| n+\alpha\left(\bar{N}_{G}\left[A_{1}\right]\right)\left|U_{1}\right| r\right)+\sum_{i=2}^{s} \alpha(G)\left|U_{i}\right| r
\end{aligned}
$$

where $\alpha\left(\bar{N}_{G}\left[A_{i}\right]\right)$ denotes the independence number of the subgraph of $G$ induced by $\bar{N}_{G}\left[A_{i}\right]$.

Now, we distinguish two cases to prove that $|S| \leq \max \{\alpha(G) \alpha(H) n, \alpha(G) r|H|\}$.

Case 1: $\frac{\alpha(H)}{|H|} \leq \frac{r}{n}$. By inequality (3.1), we have that

$$
\left|A_{1}\right|\left|U_{0}\right| n \leq\left|A_{1}\right|\left|U_{1}\right| r
$$

and so $\left|A_{1}\right|\left|U_{0}\right| n+\alpha\left(\bar{N}_{G}\left[A_{1}\right]\right)\left|U_{1}\right| r \leq\left(\left|A_{1}\right|+\alpha\left(\bar{N}_{G}\left[A_{1}\right]\right)\right)\left|U_{1}\right| r$. Then, by (3.6) and Lemma 1.3 , it follows that

$$
|S| \leq\left(\left|A_{1}\right|+\alpha\left(\bar{N}_{G}\left[A_{1}\right]\right)\right)\left|U_{1}\right| r+\sum_{i=2}^{s} \alpha(G)\left|U_{i}\right| r \leq \sum_{i=1}^{s} \alpha(G)\left|U_{i}\right| r=\alpha(G)|H| r .
$$

Case 2: $\frac{r}{n}<\frac{\alpha(H)}{|H|}$.

Subcase 2.1: $\frac{\left|U_{0}\right|}{\left|U_{1}\right|} \leq \frac{r}{n}$. By $(3.6)$ and Lemma 1.3 , we can derive that

$$
\begin{aligned}
|S| & \leq\left(\left|A_{1}\right|+\alpha\left(\bar{N}_{G}\left[A_{1}\right]\right)\right)\left|U_{1}\right| r+\sum_{i=2}^{s} \alpha(G)\left|U_{i}\right| r \\
& \leq \sum_{i=1}^{s} \alpha(G)\left|U_{i}\right| r=\alpha(G)|H| r<\alpha(G) \alpha(H) n .
\end{aligned}
$$

Subcase 2.2: $\frac{\left|U_{0}\right|}{\left|U_{1}\right|}>\frac{r}{n}$. Note that $U_{2} \cup \cdots \cup U_{s}=\bar{N}_{G}\left[U_{0}\right]$. Then by Lemma 1.3 , it follows that

$$
\left|U_{0}\right|+\frac{\alpha(H)}{|H|} \sum_{2 \leq i \leq s}\left|U_{i}\right|=\left|U_{0}\right|+\frac{\alpha(H)}{|H|}\left|\bar{N}_{H}\left[U_{0}\right]\right| \leq \alpha(H) .
$$


By (3.6), 3.9) and Lemma 1.3 , we obtain that

$$
\begin{aligned}
|S| & \leq\left(\left|A_{1}\right|\left|U_{0}\right| n+\alpha\left(\bar{N}_{G}\left[A_{1}\right]\right)\left|U_{1}\right| r\right)+\sum_{i=2}^{s} \alpha(G)\left|U_{i}\right| r \\
& \leq\left(\left|A_{1}\right|+\alpha\left(\bar{N}_{G}\left[A_{1}\right]\right)\right)\left|U_{0}\right| n+\sum_{i=2}^{s} \alpha(G)\left|U_{i}\right| r \\
& \leq \alpha(G)\left|U_{0}\right| n+\alpha(G) r \sum_{i=2}^{s}\left|U_{i}\right| \\
& \leq \alpha(G)\left|U_{0}\right| n+\alpha(G) \frac{\alpha(H)}{|H|} n \sum_{i=2}^{s}\left|U_{i}\right| \\
& =\alpha(G) n\left(\left|U_{0}\right|+\frac{\alpha(H)}{|H|} \sum_{i=2}^{s}\left|U_{i}\right|\right) \\
& \leq \alpha(G) \alpha(H) n .
\end{aligned}
$$

Therefore, $|S|=\max \{\alpha(G) \alpha(H) n, \alpha(G) r|H|\}$. Now we distinguish two cases to prove that either $T$ is regular or one of (ii) $-(\mathrm{v})$ holds.

Case 1: $T=S$.

Subcase 1.1: $U_{0}=\emptyset$. In this case, $U_{1}=\emptyset$. By the maximality of $S$ and (3.6), it follows that $|S|=\alpha(G) r|H|$. In this case, $\max \{\alpha(G) \alpha(H) n, \alpha(G) r|H|, \alpha(H) r|G|\}=\alpha(G) r|H|$, and so we have that $\min \left\{\frac{\alpha(G)}{|G|}, \frac{r}{n}\right\} \geq \frac{\alpha(H)}{|H|}$.

If $A_{i} \neq \emptyset$ for all $2 \leq i \leq s$, then the equalities in (3.4), (3.5) and (3.6) imply that $\frac{\alpha(G)}{|G|}=\frac{\alpha(H)}{|H|}$ and $A_{i}$ is imprimitive if $\bar{N}_{G}\left[A_{i}\right] \neq \emptyset$ for some $i$. That is, (iii) or (v) holds. So we suppose that $\bar{N}_{G}\left[A_{i}\right]=\emptyset$ for all $i$. Then the maximality of $S$ implies that each $A_{i}$ is a maximum independent set of $G$. In this case, $S=\left(A_{2} \times U_{2} \cup \cdots \cup A_{s} \times U_{s}\right) \times I$. If $s=2$, then $S$ is regular. Otherwise, it is easy to verify that $H$ is disconnected and one of (ii) $-(\mathrm{v})$ holds.

If $A_{i}=\emptyset$ for some $i$, then the equality in (3.4) implies that $\frac{\alpha(G)}{|G|}=\frac{\alpha(H)}{|H|}$ and $X^{\prime}=V(G)$. Thus, the equality in (3.3) implies that $B_{a}$ is a maximum independent set of $H$ or $B_{a}$ is an imprimitive set of $H$ for every $a \in V(G)$. If $B_{a}$ is a maximum independent set of $H$ every $a \in V(G)$, in the similar way, we can prove that either $S$ is regular or $G$ is disconnected; and, if $B_{a}$ is an imprimitive set of $H$ for some $a \in V(G)$, we can prove that $H$ is IS-imprimitive. That is, (iii) or (v) holds.

Subcase 1.2: $U_{0} \neq \emptyset$. When $\bar{N}_{H}\left[U_{0}\right]=\emptyset$, it is easy to see that $U_{2} \cup \cdots \cup U_{s}=\emptyset$, and the maximality of $S$ implies that $S=A_{1} \times U_{0} \times[n] \cup \bar{N}_{G}\left[A_{1}\right] \times U_{0} \times I$. If $\bar{N}_{G}\left[A_{1}\right]=\emptyset$, then $S=A_{1} \times U_{0} \times[n]$. Otherwise, $R=A_{1} \times[n] \cup \bar{N}_{G}\left[A_{1}\right] \times I$ is a maximum independent set of $G \times K_{n: r}$. Hence, the product $G \times K_{n: r}$ is not MIS-normal, and the maximality of $R$ implies $\frac{\alpha(G)}{|G|}=\frac{r}{n}=\frac{\left|A_{1}\right|}{\left|N_{G}\left[A_{1}\right]\right|}$. That is, $G$ is IS-imprimitive. By $|S|=\max \{\alpha(G) \alpha(H) n, \alpha(G) r|H|\}$, it follows that $\frac{\alpha(G)}{|G|}=\frac{\alpha(H)}{|H|}=\frac{r}{n}$. Then (v) holds. 
When $\bar{N}_{H}\left[U_{0}\right] \neq \emptyset$, if $\frac{r}{n}<\frac{\alpha(H)}{|H|}$, then $|S|=\alpha(G) \alpha(H) n$. In this case, if $\frac{\left|U_{0}\right|}{\left|U_{1}\right|} \leq \frac{r}{n}$, then it follows by (3.8) that $|S|<\alpha(G) \alpha(H) n$; and, if $\frac{\left|U_{0}\right|}{\left|U_{1}\right|}>\frac{r}{n}$, then one of the strict inequalities in 3.10 and (3.11) hold, and so $|S|<\alpha(G) \alpha(H) n$. Therefore, $\frac{r}{n} \geq \frac{\alpha(H)}{|H|}$. Then equalities in (3.1) and (3.7) imply $\frac{\alpha(H)}{|H|}=\frac{r}{n}=\frac{\left|U_{0}\right|}{\left|\bar{N}_{H}\left[U_{0}\right]\right|}$, and so $H$ is IS-imprimitive. That is, (iv) or (v) holds.

Case 2: $T \neq S$. By Case 1, it remains to verify that one of (ii)-(v) holds if $T$ is not regular but $S$ is regular. If $S=A \times U \times[n]$, it is easy to see that $T=S$, and so $T$ is also regular. Thus, we assume that $S=A \times V(H) \times I$. By the definition of $S$, it is easy to see that $S=A \times R$ for some irregular maximum independent set $R$ of $H \times K_{n: r}$. Then the direct product $H \times K_{n: r}$ is not MIS-normal. Note that $\frac{\alpha(H)}{|H|} \leq \frac{1}{2}$ and $K_{n: r}$ is IS-imprimitive or disconnected if and only if $n=2 r \geq 4$. By Theorem 1.5, either $\frac{\alpha(H)}{|H|}=\frac{r}{n}$ and one of them is IS-imprimitive or $\frac{\alpha(H)}{|H|}<\frac{r}{n}$ and $H$ is disconnected. Then (iv) or (v) holds if the former holds; and (ii) or (iii) holds if the latter holds.

Now we consider Kneser graphs and permutation graphs.

Lemma 3.1. Let $G, H$ and $K$ be three vertex-transitive graphs. If there exists an induced subgraph $K^{\prime}$ of $K$ such that $\frac{\alpha\left(K^{\prime}\right)}{\left|K^{\prime}\right|}=\frac{\alpha(K)}{|K|}$ and

$$
\alpha T\left(G, H, K^{\prime}\right)=\max \left\{\alpha(G) \alpha\left(K^{\prime}\right)|H|, \alpha(H) \alpha\left(K^{\prime}\right)|G|, \alpha(G) \alpha(H)\left|K^{\prime}\right|\right\},
$$

then

$$
\alpha T(G, H, K)=\max \{\alpha(G) \alpha(K)|H|, \alpha(H) \alpha(K)|G|, \alpha(G) \alpha(H)|K|\} .
$$

Furthermore, if $T T\left(G, H, K^{\prime}\right)$ is MIS-normal, then exactly one of the following holds:

(i) $T(G, H, K)$ is MIS-normal;

(ii) $\frac{\alpha(K)}{|K|}=\frac{\alpha(G)}{|G|}$ or $\frac{\alpha(K)}{|K|}=\frac{\alpha(H)}{|H|}$ and $K$ is imprimitive;

(iii) $\frac{\alpha(K)}{|K|}<\min \left\{\frac{\alpha(G)}{|G|}, \frac{\alpha(H)}{|H|}\right\}$ and $K$ is disconnected.

Proof. The result is obvious if $K$ is an empty graph. If one of $G$ and $H$ is an empty graph, the result holds by Theorem 1.5. So we may assume that all of them are nonempty graphs. Since $T(G, H, K)$ is a vertex-transitive graph and $T\left(G, H, K^{\prime}\right)$ is an induced subgraph of $T(G, H, K)$, by Lemma 1.3 ,

$$
\begin{aligned}
\frac{\alpha T(G, H, K)}{|T(G, H, K)|} & \leq \frac{\alpha T\left(G, H, K^{\prime}\right)}{\left|T\left(G, H, K^{\prime}\right)\right|}=\max \left\{\frac{\alpha(G) \alpha\left(K^{\prime}\right)}{|G|\left|K^{\prime}\right|}, \frac{\alpha(H) \alpha\left(K^{\prime}\right)}{|H|\left|K^{\prime}\right|}, \frac{\alpha(G) \alpha(H)}{|G||H|}\right\} \\
& =\max \left\{\frac{\alpha(G) \alpha(K)}{|G||K|}, \frac{\alpha(H) \alpha(K)}{|H||K|}, \frac{\alpha(G) \alpha(H)}{|G||H|}\right\} \leq \frac{\alpha T(G, H, K)}{|T(G, H, K)|} .
\end{aligned}
$$


Hence,

$$
\frac{\alpha T(G, H, K)}{|T(G, H, K)|}=\frac{\alpha T\left(G, H, K^{\prime}\right)}{\left|T\left(G, H, K^{\prime}\right)\right|}=\max \left\{\frac{\alpha(G) \alpha(K)}{|G||K|}, \frac{\alpha(H) \alpha(K)}{|H||K|}, \frac{\alpha(G) \alpha(H)}{|G||H|}\right\}
$$

This proves the equality in Lemma 3.1 .

For every $\sigma \in \operatorname{Aut}(K)$, where $\operatorname{Aut}(K)$ is the automorphism group of $K$, it is clear that $T\left(G, H, \sigma\left(K^{\prime}\right)\right)$ is MIS-normal. Let $S$ be a maximum independent set of $T(G, H, K)$. By Lemma 1.3 and $3.12, S \cap V T\left(G, H, \sigma\left(K^{\prime}\right)\right)$ is a maximum independent set of $T(G, H$, $\left.\sigma\left(K^{\prime}\right)\right)$. For every $k \in V(K)$, let

$$
\partial_{k}(S)=\left\{(a, u) \in \partial_{G, H}(S):(a, u, k) \in S\right\}
$$

and

$$
\partial_{K}(S)=\left\{k \in V(K): \partial_{k}(S) \neq \emptyset\right\} .
$$

For each $k \in \partial_{K}(S)$, it is clear that there is a $\sigma \in \operatorname{Aut}(K)$ such that $k \in \sigma\left(K^{\prime}\right)$. Then, by the assumption that $T\left(G, H, K^{\prime}\right)$ is MIS-normal, it follows that for $k \in \partial_{K}(S), \partial_{k}(S)=$ $A \times H$ or $G \times U$ or $A \times U$ for some $A \in I(G)$ and $U \in I(H)$. We will complete the proof in the following two cases:

Case 1: There exists an $i \in \partial_{K}(S)$ such that $\left|\partial_{i}(S)\right|=\alpha(G)|H|$ or $\alpha(H)|G|$. For symmetry, suppose $\partial_{i}(S)=A \times V(H)$ for some $i \in \partial_{K}(S)$. We now prove that for all $k \in \partial_{K}(S), \partial_{k}(S)=A \times V(H)$ or $A \times U$, where $U \in I(H)$. Suppose not, then there exists some $j \in \partial_{K}(S)$ such that $\partial_{j}(S)=B \times V(H)$ or $B \times U$ or $V(G) \times U$, where $B \in I(G)$ and $U \in I(H)$ with $A \neq B$.

If $\partial_{j}(S)=B \times V(H)$, then there exist $u$ and $v$ of $V(H)$ such that $(u, v) \in E(H)$, since $H$ is a nonempty graph. On the other hand, since $A, B \in I(G),(a, b) \in E(G)$ for some $a \in A$ and $b \in B$. Hence $(a, u, i)$ and $(b, v, j)$ are adjacent in $(G, H, K)$. However, $(a, u, i)$ and $(b, v, j)$ are both contained in the independent set $S$, yielding a contradiction.

If $\partial_{j}(S)=B \times U$, then $(a, b) \in E(G)$ for some $a \in A$ and $b \in B$ and $(u, v) \in E(H)$ for some $u \in V(H)$ and $v \in U$, since $A, B \in I(G)$ and $U \in I(H)$. So the two elements $(a, u, i)$ and $(b, v, j)$ of $S$ are adjacent in $(G, H, K)$, yielding a contradiction.

If $\partial_{j}(S)=V(G) \times U$, then there exist $a, b \in V(G)$ and $u, v \in V(H)$ such that $(a, b) \in$ $E(G),(u, v) \in E(H)$ and $a \in A, v \in U$, since $G$ and $H$ are both nonempty graphs. Hence the two elements $(a, u, i)$ and $(b, v, j)$ of $S$ are adjacent in $(G, H, K)$, yielding a contradiction.

Thus, $S=A \times R$ for some maximum independent set $R$ of $H \times K$, which implies $\frac{\alpha(G)}{|G|} \geq$ $\min \left\{\frac{\alpha(H)}{|H|}, \frac{\alpha(K)}{|K|}\right\}$. If $\partial_{k}(S)=A \times V(H)$ for all $k \in \partial_{K}(S)$, then $S=A \times V(H) \times \partial_{K}(S)$, and so $S$ is regular. Otherwise, $\partial_{i}(S)=A \times U$ for some $i \in \partial_{K}(S)$, then $R$ is irregular. By Theorem 1.5, either $\frac{\alpha(H)}{|H|}=\frac{\alpha(K)}{|K|}$ and one of them is IS-imprimitive, or $\frac{\alpha(H)}{|H|}<\frac{\alpha(K)}{|K|}$ and 
$H$ is disconnected or $\frac{\alpha(H)}{|H|}>\frac{\alpha(K)}{|K|}$ and $K$ is disconnected. However, if $\frac{\alpha(H)}{|H|}=\frac{\alpha(K)}{|K|}$ and $H$ is IS-imprimitive or $\frac{\alpha(H)}{|H|}<\frac{\alpha(K)}{|K|}$ and $H$ is disconnected, it is easy to verify that $\left(G, H, K^{\prime}\right)$ is not MIS-normal. Hence, $\frac{\alpha(H)}{|H|}=\frac{\alpha(K)}{|K|}$ and $K$ is IS-imprimitive or $\frac{\alpha(H)}{|H|}>\frac{\alpha(K)}{|K|}$ and $K$ is disconnected. That is, (ii) or (iii) holds.

Case 2: For all $k \in \partial_{K}(S),\left|\partial_{k}(S)\right|=\alpha(G) \alpha(H)$. In this case, the maximality of $S$ implies that $\frac{\alpha(K)}{|K|} \leq \min \left\{\frac{\alpha(K)}{|K|}, \frac{\alpha(H)}{|H|}\right\}$ and $\partial_{K}(S)=V(K)$. If for all $k \in V(K)$, $\partial_{k}(S)=A \times U$ for some $A \in I(G)$ and $U \in I(H)$, then $S=A \times U \times V(K)$. That is, $S$ is regular. Otherwise, we list all distinct $\partial_{k}(S)$ 's as $A_{1} \times U_{1}, \ldots, A_{s} \times U_{s}(s>2)$. For $1 \leq i \leq s$, let

$$
V_{i}=\left\{k \in V(K): \partial_{k}(S)=A_{i} \times U_{i}\right\}
$$

For any distinct pair elements $i$ and $j$ of $[s]$, since $A_{i} \times U_{i} \neq A_{j} \times U_{j}$, there exist $(a, u) \in$ $A_{i} \times U_{i}$ and $(b, v) \in A_{j} \times U_{j}$ with either $(a, b) \in E(G)$ or $(u, v) \in E(H)$. If there exist $k_{1} \in V_{i}$ and $k_{2} \in V_{j}$ with $\left(k_{1}, k_{2}\right) \in E(K)$, then the two elements $\left(a, u, k_{1}\right)$ and $\left(b, v, k_{2}\right)$ of $S$ are adjacent, yielding a contradiction. Thus, $\left(k_{1}, k_{2}\right) \notin E(H)$ for all $k_{1} \in V_{i}$ and $k_{2} \in V_{j}$. Hence $K$ is disconnected, and (iii) holds.

For the permutation group $S_{n}$, we can define a graph $G\left(S_{n}\right)$ with vertex set $S_{n}$ and two vertices $\sigma$ and $\gamma$ are adjacent if and only if $\sigma(i) \neq \gamma(i)$ for all $i \in[n]$. In [13, Wang and Zhang proved the following result.

Lemma 3.2. $K_{n, r}$ is IS-imprimitive if and only if $n=2 r \geq 4$, and $G\left(S_{n}\right)$ is ISimprimitive if and only if $n=3$.

Combing with Theorem 1.7, Lemmas 3.1 and 3.2, we have the following corollary.

Corollary 3.3. Let $G$ and $H$ be two vertex-transitive graphs with $\frac{\alpha(G)}{|G|} \geq \frac{\alpha(H)}{|H|}$. If $K$ is a Kneser graph $K_{n, r}$ or a permutation graph $G\left(S_{n}\right)$, then

$$
\alpha T(G, H, K)=\max \{\alpha(G) \alpha(H)|K|, \alpha(G) \alpha(K)|H|\}
$$

and exactly one of the following holds:

(i) $T(G, H, K)$ is MIS-normal,

(ii) $\frac{\alpha(G)}{|G|}>\frac{\alpha(K)}{|K|}=\frac{\alpha(H)}{|H|}$ and one of $H$ and $K$ is IS-imprimitive,

(iii) $\frac{\alpha(K)}{|K|}=\frac{\alpha(H)}{|H|}=\frac{\alpha(G)}{|G|}$ and one of them is IS-imprimitive,

(iv) $\min \left\{\frac{\alpha(K)}{|K|}, \frac{\alpha(G)}{|G|}\right\}>\frac{\alpha(H)}{|H|}$ and $H$ is disconnected,

(v) $\frac{\alpha(K)}{|K|}>\frac{\alpha(H)}{|H|}=\frac{\alpha(G)}{|G|}$ and one of $G$ or $H$ is IS-imprimitive. 


\section{Acknowledgments}

The authors thank Xuding Zhu for telling them the definition and problem on the tensor product of graphs.

\section{References}

[1] R. Ahlswede, H. Aydinian and L. H. Khachatrian, The intersection theorem for direct products, European J. Combin. 19 (1998), no. 6, 649-661.

[2] M. O. Albertson and K. L. Collins, Homomorphisms of 3-chromatic graphs, Discrete Math. 54 (1985), no. 2, 127-132.

[3] P. J. Cameron and C. Y. Ku, Intersecting families of permutations, European J. Combin. 24 (2003), no. 7, 881-890.

[4] P. Frankl, An Erdös-Ko-Rado theorem for direct products, European J. Combin. 17 (1996), no. 8, 727-730.

[5] X. B. Geng, J. Wang and H. J. Zhang, Structure of independent sets in direct products of some vertex-transitive graphs, Acta Math. Sin. (Engl. Ser.) 28 (2012), no. 4, 697706 .

[6] P. K. Jha and S. Klavžar, Independence in direct-product graphs, Ars Combin. 50 (1998), 53-63.

[7] G. O. H. Katona, A simple proof of the Erdös-Chao Ko-Rado theorem, J. Combinatorial Theory Ser. B 13 (1972), 183-184.

[8] C. Y. Ku and B. B. McMillan, Independent sets of maximal size in tensor powers of vertex-transitive graphs, J. Graph Theory 60 (2009), no. 4, 295-301.

[9] C. Y. Ku and T. W. H. Wong, Intersecting families in the alternating group and direct product of symmetric groups, Electron. J. Combin. 14 (2007), Research Paper 25, 15 pp.

[10] B. Larose and C. Tardif, Projectivity and independent sets in powers of graphs, J. Graph Theory 40 (2002), no. 3, 162-171.

[11] C. Tardif, Graph products and the chromatic difference sequence of vertex-transitive graphs, Discrete Math. 185 (1998), no. 1-3, 193-200.

[12] M. Valencia-Pabon and J. Vera, Independence and coloring properties of direct products of some vertex-transitive graphs, Discrete Math. 306 (2006), no. 18, 2275-2281. 
[13] J. Wang and H. Zhang, Cross-intersecting families and primitivity of symmetric systems, J. Combin. Theory Ser. A 118 (2011), no. 2, 455-462.

[14] W. Wang and Z. Yu, Independent sets in direct product of vertex-transitive graphs, Int. J. Contemp. Math. Sci. 4 (2009), no. 33-36, 1803-1808.

[15] J. Xiao, H. Zhang and S. Zhang, Fractional chromatic numbers of tensor products of three graphs, Discrete Math. 342 (2019), no. 5, 1310-1317.

[16] H. Zhang, Primitivity and independent sets in direct products of vertex-transitive graphs, J. Graph Theory 67 (2011), no. 3, 218-225.

[17] _ Independent sets in direct products of vertex-transitive graphs, J. Combin. Theory Ser. B 102 (2012), no. 3, 832-838.

[18] X. Zhu, The fractional version of Hedetniemi's conjecture is true, European J. Combin. 32 (2011), no. 7, 1168-1175.

Huiqun Mao

Department of Mathematics, Zhejiang Normal University, Jinhua 321004, China

E-mail address: 994403731@qq.com

Huajun Zhang

Department of Mathematics, Shaoxing University, Shaoxing 312000, China

E-mail address: huajunzhang@zjnu.cn 